\title{
Histologic changes and gene expression patterns in biopsy specimens from bacteria-inoculated and noninoculated excisional body and limb wounds in horses healing by second intention
}

\author{
Elin Jørgensen DVM, PhD \\ Freja B. Hjerpe DVM \\ Hans P. Hougen MD, DVM, Dr Med Sci \\ Thomas Bjarnsholt PhD, Dr Med Sci \\ Lise C. Berg DVM, PhD \\ Stine Jacobsen DVM, PhD
}

Received April I, 2019

Accepted September 5, 2019.

From the Departments of Veterinary Clinical Sciences, (Jorgensen, Hjerpe, Berg, Jacobsen), Forensic Medicine (Hougen), and Immunology and Microbiology (Bjarnsholt), Faculty of Health and Medical Sciences, University of Copenhagen, 1165 Copenhagen, Denmark; and the Department of Clinical Microbiology, Rigshospitalet, 2100 Copenhagen, Denmark (Bjarnsholt).

Address correspondence to Dr. Jørgensen (elin. jorgensen@sund.ku.dk).

\begin{abstract}
OBJECTIVE
To evaluate histologic changes and gene expression patterns in body and limb wounds in horses in response to bacterial inoculation.

SAMPLE

Wound biopsy specimens from 6 horses collected on days 7, 14, 21, and 27 after excisional wounds ( 20 wounds/horse) were created over the metacarpal and metatarsal region and lateral thoracic region (body) and then inoculated or not inoculated on day 4 with Staphylococcus aureus and Pseudomonas aeruginosa.
\end{abstract}

\section{PROCEDURES}

Specimens were histologically scored for the amount of inflammation, ede$\mathrm{ma}$, angiogenesis, fibrosis organization, and epithelialization. Quantitative PCR assays were performed to quantify gene expression of 10 inflammatory, proteolytic, fibrotic, and hypoxia-related markers involved in wound healing.

\section{RESULTS}

Except for gene expression of interleukin-6 on day 27 and tumor necrosis factor- $\alpha$ on day 14, bacterial inoculation had no significant effect on histologic scores and gene expression. Gene expression of interleukin-I $\beta$ and -6 , serum amyloid $A$, and matrix metalloproteinase- 9 was higher in limb wounds versus body wounds by day 27. Gene expression of cellular communication network factor I was higher in limb wounds versus body wounds throughout the observation period.

\section{CONCLUSIONS AND CLINICAL RELEVANCE}

The lack of clear markers of wound infection in this study reflected wellknown difficulties in detecting wound infections in horses. Changes consistent with protracted inflammation were evident in limb wounds, and gene expression patterns of limb wounds shared similarities with those of chronic wounds in humans. Cellular communication network factor warrants further investigation and may be useful in elucidating the mechanisms underlying poor limb wound healing in horses. (Am J Vet Res 2020;81:276-284)
W ound healing in mammals is a complex process, whereby several cell types are involved in a highly coordinated and complex endeavor to restore barrier function. ${ }^{1}$ The main cell types involved are keratinocytes, neutrophils, fibroblasts, macro-

$\begin{array}{ll}\text { ABBREVIATIONS } \\ \text { CCNI } & \text { Cellular communication network factor I } \\ \text { GAPDH } & \text { Glyceraldehyde 3-phosphate dehydrogenase } \\ \text { HIF } & \text { Hypoxia-inducible factor } \\ \text { IL } & \text { Interleukin } \\ \text { MCP } & \text { Monocyte chemoattractant protein } \\ \text { MMP } & \text { Matrix metalloproteinase } \\ \text { MN } & \text { Mononuclear neutrophilic leukocyte } \\ \text { PMN } & \text { Polymorphonuclear neutrophilic leukocyte } \\ \text { SAA } & \text { Serum amyloid A } \\ \text { TGF } & \text { Transforming growth factor } \\ \text { TNF } & \text { Tumor necrosis factor } \\ \text { VEGFA } & \text { Vascular endothelial growth factor A }\end{array}$

phages, endothelial cells, and platelets, and these cells communicate through and react to various signal molecules, including cytokines, growth factors, and chemokines. In well-orchestrated, normal wound healing, the wound undergoes overlapping healing phases of inflammation, proliferation, and remodeling, which are coordinated and controlled by these signal molecules. In impaired wound healing, the healing process is often halted in a sustained inflammatory phase, which has been recognized in limb wounds in horses and chronic wounds in humans. ${ }^{2,3}$

Traumatic limb wounds in horses often heal by second intention owing to tissue deficits, and these wounds often become chronic. ${ }^{4}$ Reasons for impaired limb wound healing in this species include development of exuberant granulation tissue, ,5 excessive movement, ${ }^{6}$ hypoxia,,${ }^{7,8}$ infection, ${ }^{4,9}$ and inefficient 
and prolonged inflammatory responses. ${ }^{10-12}$ In contrast, body wounds usually heal uneventfully with greater contraction, faster epithelialization, and more competent inflammation. ${ }^{10-12}$

In humans, impaired healing commonly occurs in diabetes-related foot ulcers, venous leg ulcers, and pressure sores. These wound types have different causes but share pathogenetic traits such as hypoxia and sustained chronic inflammation. ${ }^{13}$ Also common among chronic wounds in humans is the presence of biofilm infections, which is considered a major contributor to the chronicity of such wounds. ${ }^{14}$ Evidence is increasing that such biofilms play a role in impaired limb wound healing in horses as well., 15 In a recent experiment, ${ }^{16}$ we created excisional dermal limb and body wounds in horses and inoculated these wounds with Staphylococcus aureus and Pseudomonas aeruginosa on day 4 after wound creation to induce biofilm formation. Clinical findings and wound scores were similar for inoculated and noninoculated limb wounds, but from day 10 after wound creation onward, inoculated limb wounds healed slower (had larger wound areas) than did noninoculated limb wounds. Furthermore, biofilms were more often detected in inoculated limb wounds, and the majority ( $70 \%$ to $100 \%$ ) of limb wound biofilms consisted of $S$ aureus. ${ }^{16}$ On the other hand, in body wounds, the bioburden was quickly cleared and bacterial inoculation had no effect on healing. ${ }^{16}$

The purpose of the study reported here was to explore the mechanisms underlying impaired wound healing in horses after inoculation of wounds with $S$ aureus and $P$ aeruginos $a$. To do this, we used biopsy specimens from our previous study ${ }^{16}$ and assessed expression of several genes involved in healing and histologic features to further characterize the observed impaired healing. We hypothesized that the temporal expression pattern of the genes and histologic features would differ between inoculated and noninoculated limb wounds and that few or no effects of inoculation would be apparent in body wounds. A secondary hypothesis was that the inoculated limb wounds would have gene expression patterns corresponding to those found in chronic wounds in humans.

\section{Materials and Methods}

\section{Animals and specimens}

Wound biopsy specimens from 6 adult horses that participated in a previously described wound model study ${ }^{16}$ were included in the present study. In the previous study, 4 dermal wounds $(2 \times 2 \mathrm{~cm}$ in dimension and $2 \mathrm{~cm}$ apart in a vertical column) had been created in each horse by excision at 5 anatomic sites (a total of 20 wounds/horse): the dorsolateral aspect of the metatarsal region of both hind limbs and the dorsolateral aspect of the metacarpal region of 1 randomly chosen forelimb (ie, 12 limb wounds in total) and the thoracic region caudal to the shoul- der area on both sides (ie, 8 body wounds in total). On day 4 after wound creation, wounds on 2 limbs and 1 side of the thorax (12 wounds/horse) had been inoculated with $10^{4}$ CFUs of $S$ aureus and $10^{5}$ CFUs of $P$ aeruginosa. Eight wounds ( 4 limb wounds and 4 body wounds/horse) had served as noninoculated control wounds. All wounds had been bandaged with a sterile, nonadhesive gauze dressing during the observation period, and bandages had been changed every third to fourth day. On days 7, 14, 21, and 27 after wound creation, biopsy specimens had been obtained with skin biopsy punches $(6$ and $8 \mathrm{~mm})$ from 1 wound/anatomic site/horse such that each wound was biopsied only once to minimize disruption of healing; consequently, wounds were not completely healed at the completion of the study on day 27 . Sedation and analgesia had been provided to the horses prior to all procedures as described previously. ${ }^{16}$

Biopsy specimens used for histologic evaluation had been obtained from the periphery of the wound and included migrating epithelium and granulation tissue with the aim of maintaining proper orientation of the specimens and allowing evaluation of these 2 healing components. These specimens were fixed in $4 \%$ formaldehyde and embedded in paraffin; sections were cut and stained with H\&E stain. Biopsy specimens used for assessment of gene expression had been obtained from the center of the wound and included granulation tissue. These specimens had been stored directly in RNA stabilization reagent at $5^{\circ} \mathrm{C}$ for 1 day and subsequently frozen $\left(-80^{\circ} \mathrm{C}\right)$.

For the present study, biopsy specimens of intact skin from the same anatomic sites were collected once from another 6 adult research horses involved in other projects to investigate gene expression in intact skin. The experimental protocol was approved by the Danish Animal Experiments Inspectorate (1icense No. 2016-15-0201-00981) and the local ethical committee at the Department of Veterinary Clinical Sciences at the University of Copenhagen. Procedures were performed following European Union Directive 2010/63/EU for animal experiments and in accordance with the Danish Animal Testing Act.

\section{Histologic evaluation}

A certified forensic pathologist (HPH) thoroughly examined the H\&E-stained sections of wound biopsy specimens, without knowledge of anatomic location or bacterial inoculation status, and scored them using a semiquantitative grading system similar to that used in previous studies ${ }^{5,17-20}$ of contaminated and noncontaminated equine wounds. Biopsy specimens obtained on days 7, 14, 21, and 27 were subjectively scored from 0 to 3 (increasing severity) to quantify the amount of inflammatory infiltrate (on the basis of the degree of cellular invasion), edema (on the basis of the amount of separation of tissue owing to edema fluid), angiogenesis (on the basis of the amount of capillary vessels), fibrosis organization (on the basis of the apparent organization of collagen and fibro- 
blast maturation), and epithelialization (on the basis of the extent of migrating epithelium). Inflammatory infiltrate was further characterized by describing the contents of the cellular infiltrate as dominated by PMNs, dominated by MNs, or containing a mixture of PMNs and MNs. The grading was based on an overall evaluation of each slide.

\section{PCR assay analysis of gene expression}

For analysis of gene expression, biopsy specimens from intact skin and wounds were thawed, drained of stabilization reagent, and weighed. Homogenization was then performed by shredding the specimens in single-step RNA isolation reagent ${ }^{\mathrm{a}}$ in Eppendorf tubes by use of a homogenizer ${ }^{b}$ until dissolved or for a maximum of six 10-second sessions. Between shreddings, tubes containing the samples were immersed in melting ice for 20 to 30 seconds. Samples were further homogenized through shredders, ${ }^{\mathrm{c}}$ and total RNA was isolated by use of chloroform, isopropanol, and $75 \%$ ethanol in accordance with the manufacturer's protocol. ${ }^{\mathrm{d}}$ The amount and purity of total isolated RNA were measured with a spectrophotometer (260 to $280 \mathrm{~nm}){ }^{\mathrm{e}}$ Samples were kept at $-80^{\circ} \mathrm{C}$ until all samples were ready for the PCR assay.

A real-time PCR assay was performed on $1.5 \mu \mathrm{g}$ of total RNA from each sample in a total volume of 16.4 $\mu \mathrm{L}$ for each reaction. Master mix solution for each reaction contained $5.0 \mu \mathrm{L}$ of $5 \mathrm{X}$ reverse transcriptase buffer, ${ }^{\mathrm{f}} 1.3 \mu \mathrm{L}$ of dNTP, ${ }^{\mathrm{g}} 0.25 \mu \mathrm{L}$ of random hexamer primer (N6), ${ }^{\mathrm{h}} 0.25 \mu \mathrm{L}$ of oligo (dT), ${ }^{\mathrm{h}} 0.8 \mu \mathrm{L}$ of ribonuclease inhibitor, ${ }^{\mathrm{f}}$ and $1.0 \mu \mathrm{L}$ of Moloney murine leukemia virus enzyme. ${ }^{\mathrm{f}}$ The reverse transcriptase reaction was performed with a thermocycler, ${ }^{\mathrm{i}}$ and the produced cDNA samples were kept at $-20^{\circ} \mathrm{C}$ until further analysis.

Intron-spanning equine primers were used to amplify the genes (Appendix) in a quantitative realtime PCR assay performed with a cyanine dye-containing master mix ${ }^{j}$ and a real-time PCR assay system. ${ }^{k}$ All samples were prepared and run as triplicates, and each quantitative PCR assay plate contained a positive control substance (calibrator) and negative control substance (double-distilled water). Results were calculated by use of the $2^{-\Delta \Delta \mathrm{Ct}}$ method (where $\mathrm{Ct}=$ cycle threshold) and reported as relative expression ratios to the reference gene GAPDH. ${ }^{21}$ Melting curve analyses were performed routinely to validate specificity of the quantitative PCR assay products.

\section{Statistical analysis}

Histologic scores were analyzed as ordinal and categorical data. Distributions of these scores per time point were evaluated by means of the Fisher exact test, comparing body versus limb wounds and inoculated versus noninoculated wounds. Values for gene expression were compared between groups by means of 2-way ANOVA controlling for repeated measurements, in which time and wound type (limb vs body wound and inoculated vs noninoculated) were treated as fixed effects and horse and anatomic site within horse were treated as random effects. Comparisons of gene expression in biopsy specimens from intact body and limb skin were performed with the Student $t$ test. To achieve variance homogeneity of residuals and satisfactory model check results, normalized gene expression ratios were logarithmically transformed. Values of $P<0.05$ were considered significant. Computer software was used for statistical analyse $^{1}$ and generation of graphs. ${ }^{\mathrm{m}}$

\section{Results}

\section{Histologic evaluation}

On day 7 after wound creation, most wounds (50\% to $83 \%$, depending on the wound type) had histologic findings indicative of acute inflammation dominated by PMNs (Table I). Body wounds progressed through the inflammatory phase faster than did limb wounds, as MNs appeared earlier and to a greater extent in the cellular infiltrate in body wounds than in limb wounds; however, this difference was not significant. Polymorphonuclear leukocytes appeared to dominate the inflammation for longer in inoculated versus noninoculated limb wounds, but not significantly (eg, $P=0.24$ on day 21) so. Limb wounds persisted in the acute inflammatory phases (ie, PMNs or both PMNs and MNs dominated) for a longer period than did body wounds

Table I-Distributions of dominant cellular infiltrate types in histologically evaluated wound biopsy specimens collected from 6 horses at various points after excisional dermal wounds were created in the metacarpal and metatarsal region (limb) and lateral thoracic region (body) and then inoculated or not inoculated 4 days later (day 4) with Staphylococcus aureus and Pseudomonas aeruginosa.

\begin{tabular}{|c|c|c|c|c|c|c|c|c|c|c|c|c|c|c|c|c|}
\hline \multirow[b]{2}{*}{$\begin{array}{l}\text { Inoculation } \\
\text { status }\end{array}$} & \multicolumn{4}{|c|}{ Day 7} & \multicolumn{4}{|c|}{ Day I4 } & \multicolumn{4}{|c|}{ Day 21} & \multicolumn{4}{|c|}{ Day 27} \\
\hline & PMN & $\begin{array}{l}\text { MN+ } \\
\text { PMN }\end{array}$ & $\mathbf{M N}$ & NI & PMN & $\begin{array}{l}\text { MN+ } \\
\text { PMN }\end{array}$ & MN & NI & PMN & $\begin{array}{l}\text { MN+ } \\
\text { PMN }\end{array}$ & MN & NI & PMN & $\begin{array}{l}\text { MN+ } \\
\text { PMN }\end{array}$ & MN & NI \\
\hline \multicolumn{17}{|l|}{ Limb } \\
\hline Noninoculated $(n=6)$ & $4(67)$ & $2(33)$ & $0(0)$ & $0(0)$ & $3(50)$ & $2(33)$ & I (I7) & $0(0)$ & I (I7) & $4(67)$ & I $(17$ & $0(0)$ & $2(33)$ & $2(33)$ & $2(33)$ & $0(0)$ \\
\hline Inoculated* & $10(83)$ & $2(17)$ & $0(0)$ & $0(0)$ & $7(64)$ & $4(36)$ & $0(0)$ & $0(0)$ & $7(58)$ & $4(33)$ & $0(0)$ & I (8) & I (17) & $3(50)$ & $2(33)$ & $0(0)$ \\
\hline \multicolumn{17}{|l|}{ Body } \\
\hline Noninoculated $(n=6)$ & $5(83)$ & $\mathrm{I}(\mathrm{I7})$ & $0(0)$ & $0(0)$ & $2(33)$ & $3(50)$ & $\mathrm{I}(17)$ & $0(0)$ & $\mathrm{I}(\mathrm{I7})$ & $2(33)$ & $2(33)$ & I (I7) & $0(0)$ & I (I7) & $3(50)$ & $2(33)$ \\
\hline Inoculated $(n=6)$ & $3(50)$ & $3(50)$ & $0(0)$ & $0(0)$ & $3(50)$ & $3(50)$ & $0(0)$ & $0(0)$ & I (17) & $2(33)$ & $2(33)$ & I (I7) & $0(0)$ & $0(0)$ & $3(50)$ & $3(50)$ \\
\hline
\end{tabular}

Values represent the number (\%) of all specimens that contained the indicated dominant cell type.

*Number of wounds ranged from 12 on day 7 to 6 on day 27.

$\mathrm{MN}+\mathrm{PMN}=$ Cellular infiltrate with equal distributions of MNs and PMNs. NI = No inflammatory infiltrate. 

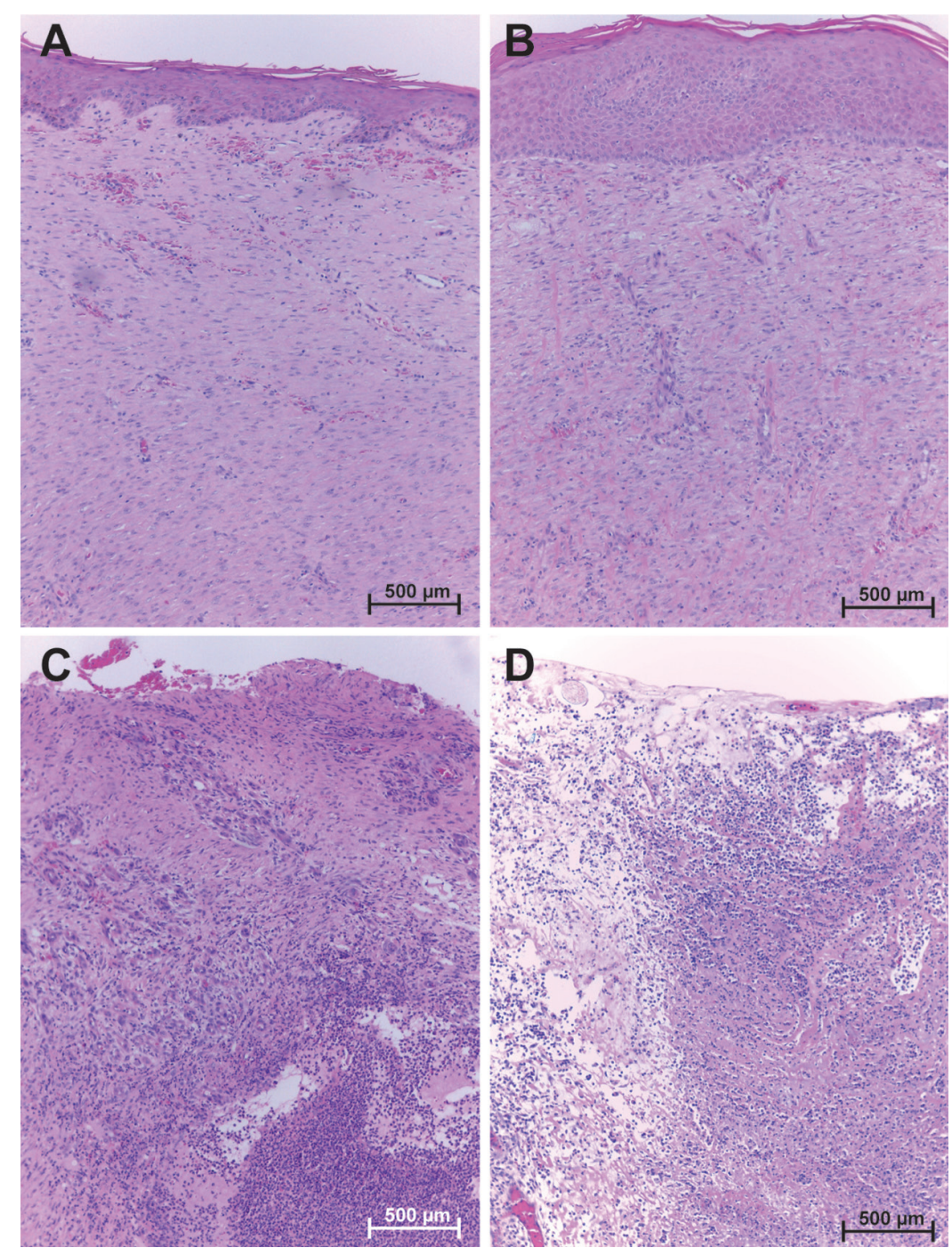

Figure I-Representative photomicrographs of biopsy specimens from excisional wounds created on the body (over the thorax; $A$ and $B$ ) and limbs (over the metatarsal and metacarpal region; $C$ and D) in horses. Four days after creation, some of the wounds were inoculated with Staphylococcus aureus and Pseudomonas aeruginosa. All wound biopsy specimens were histologically scored on a scale of 0 to 3 for the amount of inflammation, edema, angiogenesis, fibrosis organization, and epithelialization. A-Inoculated body wound on day 27 , showing mild inflammation (score $=\mathrm{I}$; dominated by MNs) and edema (score $=\mathrm{I}$ ), ongoing moderate angiogenesis (score $=2$ ), reepithelialization (score $=2$ ), and mild fibrosis organization (score $=\mathrm{I}$ ). B-Noninoculated body wound on day $2 \mathrm{I}$, showing moderate fibrosis organization (score $=2$ ), mild angiogenesis (score $=1$ ), resolved inflammation (score $=0)$, and marked reepithelialization (score $=3)$. C-Inoculated limb wound on day 21 showing moderate inflammation (score $=2$; dominated by $\mathrm{PMNs}$ ), edema (score $=2$ ), and angiogenesis (score $=2)$. D-Inoculated limb wound on day 7 , showing marked inflammation (score $=3$; dominated by PMNs) and moderate edema (score $=2)$. H\&E stain; bar $=500 \mu \mathrm{m}$.

$(P=0.008$ on day 27$)$. Histologic scores for inflammatory infiltrate were significantly $(P=0.04)$ higher in limb wounds versus body wounds on day 27 , whereas bacterial inoculation had no effect on these scores in both limb and body wounds (Supplementary Table SI, available at avmajournals.avma.org/doi/suppl/10.2460/ ajvr.81.3.276).

Photomicrographs of representative wound biopsy specimens were obtained (Figure I). From day 14 onward, limb wounds had significantly $(P=0.02$ on day $14, P=$ 0.007 on day 21 , and $P=0.006$ on day 27) higher scores for edema than did body wounds; no significant effect of inoculation was detected (Supplementary Table S2, available at avmajournals.avma.org/doi/suppl/10.2460/ ajvr.81.3.276). Limb wounds had significantly higher angiogenesis scores than did body wounds on days $7(P=$ $0.0498)$ and $21(P=0.045)$, but not on days $14(P=0.31)$ or $27(P=0.10)$; no significant effect of inoculation was detected (Supplementary Table S3, available at avmajournals.avma.org/ doi/suppl/10.2460/ajvr.81.3.276).

The amount of fibroblast organization in body wounds was superior (reflected by higher scores) to that in limb wounds on days $14(P=0.02)$ and $27(P$ $=0.02$ ) but was unaffected by inoculation (Supplementary Table S4, available at avmajournals.avma.org/doi/suppl/10.2460/ajvr.81.3.276). Body wounds epithelialized faster than did limb wounds, as reflected by higher scores from day 7 onward $(P=0.056$ on day 7 , $P=0.04$ on day $14, P=0.17$ on day 21 , and $P=0.009$ on day 27); again, no significant effect of bacterial inoculation was detected (Supplementary Table S5, available at avmajournals.avma.org/ doi/suppl/10.2460/ajvr.81.3.276).

\section{Gene expression}

Bacterial inoculation had minimal effects on expression patterns of genes associated with wound healing in the 6 horses; gene expression of IL-6 in inoculated limb wounds was 4.6 times that in noninoculated limb wounds on day $27(P=0.02)$; gene expression of TNF- $\alpha$ was 2.0 times that in inoculated versus noninoculated body wounds on day $14(P=0.04)$. Gene expression of IL-1 $\beta$, IL-6, SAA, and MMP-9 differed between limb and body wounds over time, with limb wounds having significantly higher expression than did body wounds; these differences were most pronounced in the late phases of healing toward the end of the observation period (Figure 2). Gene expression of MCP-1 also differed between limb and body wounds over time, with body wounds having significantly $(P<0.001)$ higher expression on day 7 and limb wounds having significantly $(P=0.03)$ higher expression on day 14. Gene expression of CCN1 was persistently higher in limb wounds than in body wounds throughout the entire observation period $(P$ 
$<0.001)$. For HIF- $1 \alpha$, TGF- $\beta_{1}$, VEGFA, and TNF- $\alpha$, no differences were observed in gene expression pat-
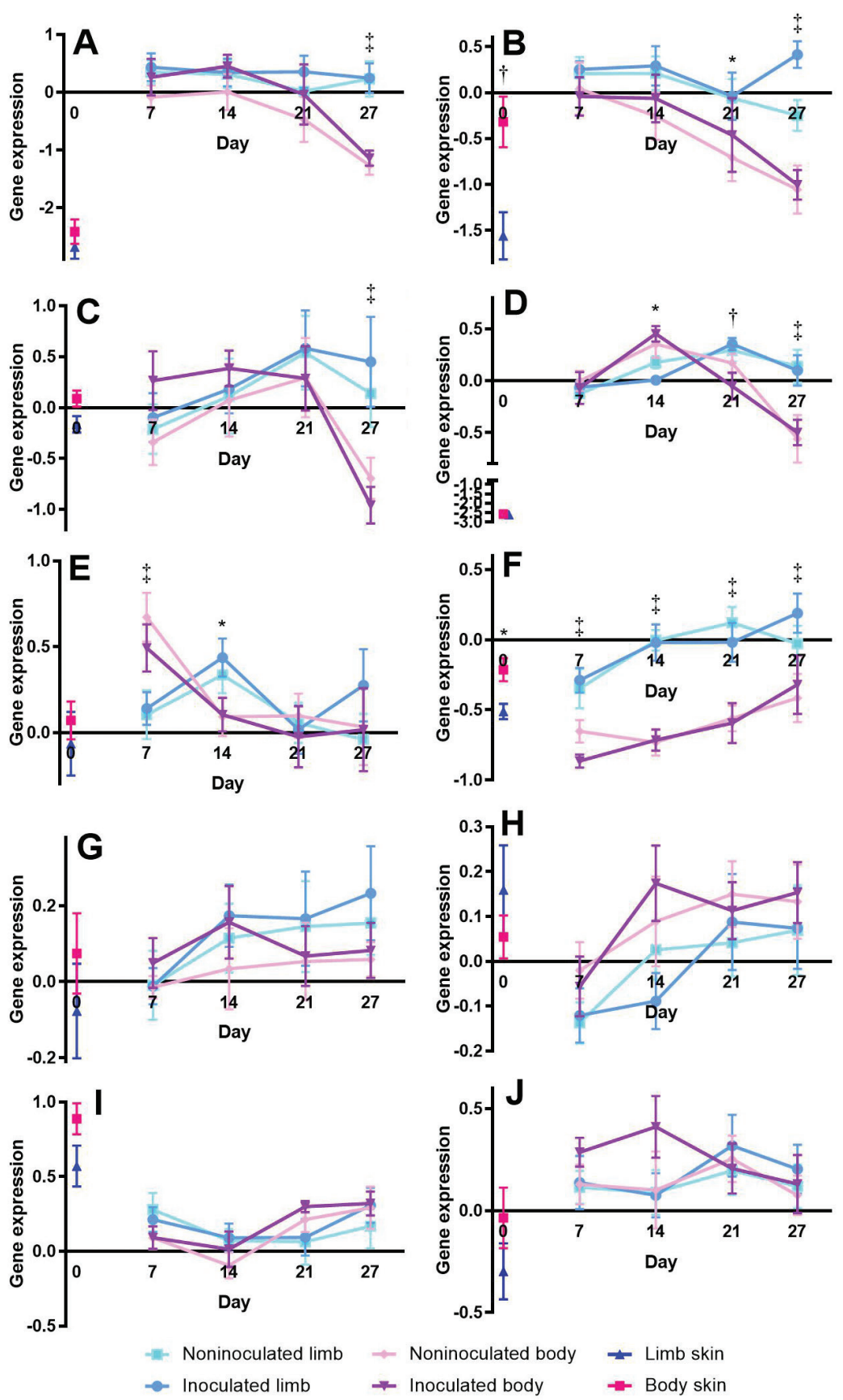

Figure 2-Expression of genes for IL-I $\beta$ (A), IL-6 (B), SAA (C), MMP-9 (D) MCP-I (E), CCNI (F), HIF-I $\alpha(G)$, TGF- $\beta I(H)$, VEGFA (I), and TNF- $\alpha(J)$ in biopsy specimens collected from 6 horses at various points after excisional wounds were created in the metacarpal and metatarsal region (limb; squares and circles) and lateral thoracic region (body; diamonds and inverted triangles) and then inoculated (circles and inverted triangles) or not inoculated (squares and diamonds) 4 days later (day 4) with $S$ aureus and $P$ aeruginosa as well as in specimens collected from intact limb (upright triangles) or body (rectangles) skin (day 0) of 6 other horses. Data points represent mean logarithmically transformed values of ratios normalized to GAPDH expression ( $n=6 /$ measurement point), and error bars represent the SEM of those values. $* \dagger \ddagger A t$ the indicated measurement point, values differ significantly ${ }^{*} P<0.05$, $+P<0.01$, and $\ddagger P<0.001$ ) between limb and body wounds (without consideration of inoculation status) or limb and body skin. Significant differences were also identified between limb and body wounds over time for IL-I $\beta(P<0.0 \mathrm{I})$, IL-6 $(P<0.00 \mathrm{I})$, SAA $(P<0.0 \mathrm{I})$, MMP-9 $(P<0.00 \mathrm{I})$, and MCP-I $(P<0.00 \mathrm{I})$. terns between limb and body wounds over time.

pecimens of intact skin from 6 other horses, gene expression of IL- 6 and CCN1 was significantly ( $P=0.01$ for both comparisons) higher in body skin than in limb skin (Figure 2).

\section{Discussion}

Limb and body wounds in the horses of the present study had pronounced differences in histologic features and gene expression levels, whereas bacteria-inoculated and noninoculated wounds had fewer differences in such characteristics. These results therefore supported previously reported evidence of differences in the propensity for healing between limb and body wounds in horses,, 922 but they did not shed further light on the mechanisms underlying our previous observation that inoculated limb wounds with biofilm formation healed slower than did noninoculated limb wounds. ${ }^{16}$

The effects of bacterial inoculation on histologic findings for equine wounds appeared to be minimal. In the present study, excisional wounds were inoculated with $S$ aureus and $P$ aeruginosa 4 days after creation, unlike accidental wounds that become contaminated immediately; however, similar to the results of the present study, no significant differences in histologic scores were identified between fecescontaminated equine limb wounds and noncontaminated wounds in 2 other experimental studies. ${ }^{17,23}$ Wounds harboring $P$ aeruginos $a$ biofilms are known from both murine and human wound studies $^{24,25}$ to contain greater numbers of PMNs, compared with wounds harboring $S$ aureus biofilms. As shown in our previous study, ${ }^{16}$ mainly $S$ aureus biofilms had formed in the wounds investigated in the present study, which might explain the lack of significantly increased inflammation scores for inoculated wounds.

The inflammatory response to biofilm infections has been found to be weak and prolonged, compared with the response associated with acute planktonic infections. ${ }^{26}$ In an experiment involving rabbits, ${ }^{26}$ gene expression of IL-1 $\beta$ and TNF- $\alpha$ was markedly higher in acute $S$ aureus planktonic ear wound infections than in biofilm ear wound infections, and gene expression of TNF- $\alpha$ did not differ between biofilm-infected 
wounds and control wounds. This corresponds with the findings of the present study, in which few differences in gene expression patterns were detected between inoculated and noninoculated wounds. The higher expression levels for the proinflammatory cytokine IL- 6 identified on day 27 in the biopsy specimens from inoculated limb wounds might have reflected a prolonged inflammatory response to the inoculation. Similarly, gene expression of IL- 6 was also increased after 4 weeks in an experiment ${ }^{27}$ involving $P$ aeruginosa biofilm-infected wounds in mice, compared with expression in control wounds. ${ }^{27}$

In the present study, the only detected effect of bacterial inoculation of body wounds was increased gene expression of TNF- $\alpha$ on days 7 and 14, compared with expression in noninoculated wounds on those days. Body wounds in horses have a more efficient and stronger inflammatory response than do limb wounds, ${ }^{10,11}$ and the early peak in expression for TNF- $\alpha$ after inoculation of body wounds could have reflected a sound (potent and short-lived) innate immune response taking place in response to the presence of bacteria and potentially aiding in clearance of the infection. Gene expression patterns of cytokines in experiments involving biofilminfected wounds appear to differ by animal species, wound type, bacterial species, and study design. However, in biofilm-infected wounds in pigs $^{28}$ and rabbits, ${ }^{29}$ gene expression of TNF- $\alpha$ also increases in response to infection.

Several markers of wound healing were unaffected by bacterial inoculation in the study reported here. Prior to our study, gene expression of MCP-1 had not been previously investigated in relation to wound inoculations in horses, and our findings indicated that such expression was unaffected by bacterial inoculation in both limb and body wounds. Likewise, there was no effect of inoculation on findings for MMP-9. Similar to these results, a study ${ }^{30}$ involving humans revealed comparable gene expression of MMP-9 in infected and noninfected venous leg ulcers. The amount of SAA in circulation increases dramatically in relation to systemic inflammation and infections in horses. ${ }^{31}$ However, to our knowledge, this reactant has not been investigated in relation to wound inoculations before. In the present study, gene expression of SAA did not differ between inoculated and noninoculated wounds for both limb and body wounds. Given that systemic SAA concentration mainly increases in relation to inflammation, ${ }^{31}$ this lack of increased gene expression in inoculated wounds might have been attributable to the inoculation not having resulted in a markedly altered or increased amount of inflammation in the wounds, as also indicated by expression levels of the other investigated inflammatory gene markers. Similar to findings for mice with $P$ aeruginos $a$-inoculated wounds, ${ }^{27}$ gene expression levels of the profibrotic and proinflammatory cytokines TGF- $\beta 1$, HIF- $1 \alpha$, and VEGFA in inoculated and noninoculated wounds did not differ.
Generally, it is difficult to detect whether and when bacteria and infection affect healing because no simple markers exist to quickly assess wound healing, whether clinically or nonclinically. ${ }^{14,26}$ The lack of clear histologic and gene expression patterns for inoculated versus noninoculated limb wounds in the present study emphasized the well-known problems in diagnosing relevant biofilm wound infections. ${ }^{14}$ Overall, few differences in histologic scores and gene expression levels were identified between inoculated and noninoculated wounds. This may have been because no such link to the selected markers exists and because biofilms induce low-grade inflammatory responses, compared with responses associated with acute planktonic infections. ${ }^{26}$ A more severe wound model, including higher inoculation doses and more pronounced acute infection, might have revealed greater differences between inoculated and noninoculated wounds. However, we were reluctant to use higher CFUs of bacteria for inoculation given the results of our initial study. In addition, we aimed to establish the type of low-grade infection that we typically see in equine limb wounds that heal by second intention.

A limitation of the study reported here was the small sample size ( 6 horses with a total of 120 wounds); this sample size had been calculated on the basis of expected differences in healing of inoculated and noninoculated wounds. ${ }^{16}$ The gene expression levels differed substantially among individual horses, and additional research with larger sample sizes is needed to better understand how bacterial inoculation and infection affect wound healing in horses. Another limitation was that results of quantitative PCR assays only reveal protein regulation at the transcriptional level and not posttranscriptional regulation, translational regulation, or protein degradation regulation. Had the investigated genes been assessed at the protein level, the results could have been different, and combining these methods in future studies could further improve our understanding. An important limitation of wounds created by excision in experimental settings is that such wounds may have better healing potential than accidental wounds. Indeed, accidental wounds often have some degree of tissue contusion and contamination as well as exposed underlying bone, ${ }^{4,32}$ which we suspect would affect healing.

The role of CCN1 in wound healing has not been explored in horses before, to our knowledge. Gene expression levels of this marker were unaffected by bacterial inoculation but were significantly higher in limb wounds than in body wounds throughout the observation period. Studies in rodents and humans have shown that CCN1 is involved in several processes relevant to wound healing, such as removal and phagocytosis of neutrophils by macrophages, ${ }^{33}$ senescence of myofibroblasts, ${ }^{34}$ and induction of angiogenesis. ${ }^{35}$ In chronic wounds in humans, gene expression of CCN1 is greater than that in intact skin, ${ }^{36}$ similar to our findings for horses during the observation period. 
During the inflammatory phase, $\mathrm{CCN} 1$ promotes the removal of neutrophils through macrophage phagocytosis to minimize neutrophil apoptosis and apoptosis-derived inflammation. ${ }^{33}$ The prolonged PMN-dominated inflammatory phase in the equine limb wounds of the present study might have induced the increase in gene expression observed for CCN1 between days 7 and 14. Because CCN1 also induces angiogenesis, ${ }^{35}$ its increased gene expression levels in limb wounds could have been linked to the higher angiogenesis scores for these wounds.

A second rise in CCN1 concentration is expected during the remodeling phase of wound healing, when CCN1 induces myofibroblast senescence, thereby turning extracellular matrix-producing myofibroblasts into antifibrotic, extracellular matrix-degrading, senescent cells. ${ }^{34}$ In the body wounds of the present study, gene expression of CCN1 increased throughout the observation period, and this increase could have been related to the ongoing remodeling phase. Lack of CCN1 in mice reportedly results in exacerbated fibrosis. ${ }^{37}$ Whether the lack of a second rise in CCN1 gene expression in equine limb wounds was related to the formation of exuberant granulation tissue, hypertrophic scarring, or protracted healing is unknown. Given our findings, additional research is warranted into the role of CCN1 in wound healing in horses. Indeed, knowledge of the exact role of CCN1 in equine wound healing might be important to understand the differences between the divergent healing patterns of limb and body wounds in horses.

The well-established differences in healing patterns of limb and body wounds in horses were supported by our findings; slower and less organized limb wound healing was shown histologically, ${ }^{, 51,19}$ and the gene expression patterns reflected the prolonged and more chronic inflammatory response characteristic of limb versus body wounds. ${ }^{10,11}$ The delayed inflammatory response of limb wounds was evident in the delayed peak in MCP-1 gene expression of limb wounds (day 14) versus body wounds (day 7), as has also been reported for wounds in diabetic versus nondiabetic mice. ${ }^{38}$ Further, gene expression of the inflammatory markers SAA, IL-1 $\beta$, and IL- 6 remained higher in limb wounds than in body wounds toward the end of the observation period and appeared to be linked to poor healing. This pattern for limb wounds shares many similarities with the pattern for chronic wounds in humans, in whom expression of IL-1 $\beta$, IL-6, MMP-9, and CCN1 also increases. ${ }^{36,39-41}$

\section{Acknowledgments}

This manuscript represents a portion of a thesis submitted by Dr. Jørgensen to the University of Copenhagen as partial fulfillment of the requirements for a Doctor of Philosophy degree.

Funded by the Horse Levy Foundation, Toosbuy Foundation, and Carl and Ellen Hertz' Foundation. The funding sources had no involvement in the data collection, data analyses, or the preparation of the manuscript.

Dr. Jørgensen's work was funded by a Danish Government PhD grant. Dr. Bjarnsholt's work was funded by the Lundbeck Foundation.
The authors declare that there were no conflicts of interest.

The authors thank Tina Roust and Maria Rhod for practical laboratory assistance.

\section{Footnotes}

a. TRI reagent, Nordic Diagnostica AB, Billdal, Norway.

b. IKA 1000 homogenizer, IKA-Werke GmbH \& Co. KG, Staufen, Germany.

c. QIAshredders, QIAGEN Danmark, Copenhagen, Denmark

d. Molecular Research Center Inc, Cincinnati, Ohio.

e. NanoDrop 2000 spectrophotometer, Fisher Scientific, Slangerup, Denmark.

f. Promega Biotech AB, Nacka, Sweden

g. InvitrogenTM, Fisher Scientific, Slangerup, Denmark.

h. TAG Copenhagen, Frederiksberg, Denmark.

i. Biometra T-personal 48 thermocycler, Analytik Jena AG, Jena, Germany.

j. LightCycler 480 SYBR green I master mix, Roche Diagnostics A/S, Hvidovre, Denmark.

k. LightCycler 480 real-time PCR System, Roche Diagnostics A/S, Hvidovre, Denmark

1. SAS Enterprise Guide, version 7.1, SAS Institute Inc, Cary, NC.

m. GraphPad Prism 5, GraphPad Software, La Jolla, Calif.

\section{References}

1. Borena BM, Martens A, Broeckx SY, et al. Regenerative skin wound healing in mammals: state-of-the-art on growth factor and stem cell based treatments. Cell Physiol Biochem 2015;36:1-23.

2. Theoret C. Physiology of wound healing. In: Theoret C, Schumacher J, eds. Equine wound management. 3rd ed. Ames, Iowa: John Wiley \& Sons Inc, 2017;1-13.

3. Grice EA, Segre JA. Interaction of microbiome and the innate immune response in chronic wounds. Adv Exp Med Biol 2012;946:55-68.

4. Maher M, Kuebelbeck L. Nonhealing wounds of the equine limb. Vet Clin North Am Equine Pract 2018;34:539-555.

5. Lepault E, Celeste C, Dore $\mathrm{M}$, et al. Comparative study on microvascular occlusion and apoptosis in body and limb wounds in the horse. Wound Repair Regen 2005;13:520529.

6. Adam EN, Southwood LL. Surgical and traumatic wound infections, cellulitis, and myositis in horses. Vet Clin North Am Equine 2006;22:335-361.

7. Sørensen MA, Pedersen LJ, Bundgaard L, et al. Regional disturbances in metabolism and blood flow in equine limb wounds healing with formation of exuberant granulation tissue. Wound Repair Regen 2014;22:647-653

8. Celeste CJ, Deschene K, Riley CB, et al. Regional differences in wound oxygenation during normal healing in an equine model of cutaneous fibroproliferative disorder. Wound Repair Regen 2011;19:89-97.

9. Jørgensen E, Bay L, Bjarnsholt T, et al. The occurrence of biofilm in an equine experimental wound model of healing by secondary intention. Vet Microbiol 2017;204:90-95.

10. Bundgaard L, Bendixen E, Sorensen MA, et al. A selected reaction monitoring-based analysis of acute phase proteins in interstitial fluids from experimental equine wounds healing by secondary intention. Wound Repair Regen 2016;24:525532.

11. Wilmink JM, Van Weeren PR, Stolk PWT, et al. Differences in second-intention wound healing between horses and ponies: histological aspects. Equine Vet J 1999;31:61-67.

12. Wilmink JM, Veenman JN, van den Boom R, et al. Differences in polymorphonucleocyte function and local inflammatory response between horses and ponies. Equine Vet J 2003;35:561-569.

13. Mustoe T. Understanding chronic wounds: a unifying hypothesis on their pathogenesis and implications for therapy. Am J Surg 2004;187:65S-70S.

14. Schultz G, Bjarnsholt T, James GA, et al. Consensus guidelines for the identification and treatment of biofilms in chron- 
ic nonhealing wounds. Wound Repair Regen 2017;25:744757.

15. Freeman $\mathrm{K}$, Woods E, Welsby $\mathrm{S}$, et al. Biofilm evidence and the microbial diversity of horse wounds. Can J Microbiol 2009;55:197-202.

16. Jørgensen E, Bay L, Skovgaard LT, et al. An equine wound model to study effects of bacterial aggregates on wound healing. Adv Wound Care (New Rochelle) 2019;8:487-498

17. Bischofberger AS, Dart CM, Horadagoda N, et al. Effect of Manuka honey gel on the transforming growth factor $\beta$ and $\beta 3$ concentrations, bacterial counts and histomorphology of contaminated full-thickness skin wounds in equine distal limbs. Aust Vet J 2016;94:27-34.

18. Harmon CCG, Hawkins JF, Li J, et al. Effects of topical application of silver sulfadiazine cream, triple antimicrobial ointment, or hyperosmolar nanoemulsion on wound healing, bacterial load, and exuberant granulation tissue formation in bandaged full-thickness equine skin wounds. Am J Vet Res 2017;78:638-646.

19. Theoret CL, Barber SM, Moyana TN, et al. Expression of transforming growth factor $\beta 1, \beta 3$, and basic fibroblast growth factor in full-thickness skin wounds of equine limbs and thorax. Vet Surg 2001;30:269-277.

20. Theoret CL, Olutoye OO, Parnell LKS, et al. Equine exuberant granulation tissue and human keloids: a comparative histopathologic study. Vet Surg 2013;42:783-789.

21. Livak KJ, Schmittgen TD. Analysis of relative gene expression data using real-time quantitative PCR and the 2(-delta delta $\mathrm{C}(\mathrm{T}))$ method. Methods 2001;25:402-408.

22. Wilmink JM, Stolk PWT, Van Weeren PR, et al. Differences in second-intention wound healing between horses and ponies: macroscopic aspects. Equine Vet J 1999;31:53-60.

23. Bischofberger AS, Dart CM, Perkins NR, et al. The effect of short- and long-term treatment with Manuka honey on second intention healing of contaminated and noncontaminated wounds on the distal aspect of the forelimbs in horses. Vet Surg 2013;42:154-160.

24. Fazli M, Bjarnsholt T, Kirketerp-Møller K, et al. Quantitative analysis of the cellular inflammatory response against biofilm bacteria in chronic wounds. Wound Repair Regen 2011;19:387-391.

25. Trøstrup H, Thomsen K, Christophersen LJ, et al. Pseudomonas aeruginosa biofilm aggravates skin inflammatory response in BALB/c mice in a novel chronic wound model. Wound Repair Regen 2013;21:292-299.

26. Gurjala AN, Geringer MR, Seth AK, et al. Development of a novel, highly quantitative in vivo model for the study of biofilm-impaired cutaneous wound healing. Wound Repair Regen 2011;19:400-410.

27. Zhao G, Usui ML, Underwood RA, et al. Time course study of delayed wound healing in a biofilm-challenged diabetic mouse model. Wound Repair Regen 2012;20:342-352.

28. Pastar I, Nusbaum AG, Gil J, et al. Interactions of methicillin resistant Staphylococcus aureus USA300 and Pseudomonas aeruginosa in polymicrobial wound infection. PLoS One 2013;8:e56846.
29. Seth AK, Geringer MR, Hong SJ, et al. Comparative analysis of single-species and polybacterial wound biofilms using a quantitative, in vivo, rabbit ear model. PLoS One 2012;7:e42897.

30. Serra R, Grande R, Buffone G, et al. Extracellular matrix assessment of infected chronic venous leg ulcers: role of metalloproteinases and inflammatory cytokines. Int Wound $J$ 2016;13:53-58.

31. Jacobsen SA, Andersen PH. The acute phase protein serum amyloid A (SAA) as a marker of inflammation in horses. Equine Vet Educ 2007;19:38-46.

32. Wilmink JM, van Herten J, van Weeren PR, et al. Retrospective study of primary intention healing and sequestrum formation in horses compared to ponies under clinical circumstances. Equine Vet J 2002;34:270-273.

33. Jun JI, Kim KH, Lau LF. The matricellular protein CCN1 mediates neutrophil efferocytosis in cutaneous wound healing. Nat Commun 2015;6:7386.

34. Jun JI, Lau LF. Resolution of organ fibrosis. J Clin Invest 2018;128:97-107.

35. Babic AM, Kireeva ML, Kolesnikova TV, et al. CYR61, a product of a growth factor-inducible immediate early gene, promotes angiogenesis and tumor growth. Proc Natl Acad Sci U $S$ A 1998;95:6355-6360.

36. Roy S, Patel D, Khanna S, et al. Transcriptome-wide analysis of blood vessels laser captured from human skin and chronic wound-edge tissue. Proc Natl Acad Sci U S A 2007; 104:14472-14477.

37. Jun JI, Lau LF. The matricellular protein CCN1 induces fibroblast senescence and restricts fibrosis in cutaneous wound healing. Nat Cell Biol 2010;12:676-685.

38. Wetzler C, Kampfer H, Stallmeyer B, et al. Large and sustained induction of chemokines during impaired wound healing in the genetically diabetic mouse: prolonged persistence of neutrophils and macrophages during the late phase of repair. J Invest Dermatol 2000;115:245-253.

39. Karam RA, Rezk NA, Abdel Rahman TM, et al. Effect of negative pressure wound therapy on molecular markers in diabetic foot ulcers. Gene 2018;667:56-61.

40. Jiang L, Dai Y, Cui F, et al. Expression of cytokines, growth factors and apoptosis-related signal molecules in chronic pressure ulcer wounds healing. Spinal Cord 2014;52:145-151.

41. Wang Y, Dai YL, Piao JL, et al. The expressions and functions of inflammatory cytokines, growth factors and apoptosis factors in the late stage of pressure ulcer chronic wounds [in Chinese]. Zhongguo Ying Yong Sheng Li Xue Za Zbi 2017;33:181-184.

42. Iqbal J, Bird JL, Hollander AP, et al. Effect of matrix depleting agents on the expression of chondrocyte metabolism by equine chondrocytes. Res Vet Sci 2004;77:249-256.

43. Deschene K, Céleste C, Boerboom D, et al. Constitutive expression of hypoxia-inducible factor-1 $\alpha$ in keratinocytes during the repair of skin wounds in horses. Wound Repair Regen 2011;19:250-259.

44. Dahlgren LA, Mohammed HO, Nixon AJ. Temporal expression of growth factors and matrix molecules in healing tendon lesions. J Orthop Res 2005;23:84-92. 


\section{Appendix}

Sequences of primers* used for quantitative PCR assays of expression of genes associated with markers of wound healing in equine wound and skin biopsy specimens.

\begin{tabular}{|c|c|c|c|}
\hline Gene or marker & Forward primer sequence $\left(5^{\prime}\right.$ to $\left.3^{\prime}\right)$ & Reverse primer sequence $\left(5^{\prime}\right.$ to $\left.3^{\prime}\right)$ & GenBank accession No. \\
\hline GAPDH & GGG TGG AGC CAA AAG GGT CAT CAT & AGC TTT CTC CAG GCG GCA GGT CAG & NM_00II63856.I \\
\hline IL-I $\beta$ & GGC ATC CAG CTT CAA TTC TC & ACA GCA CCA GGG ATT TAT GG & NM_001082526.I \\
\hline IL-6 & ATG GCA GAA AAA GAC GGA TG & GGG TCA GGG GTG GTT ACT TC & NM_001082526. \\
\hline MMP-9 & GAG ATC GGG AAT CAT CTC CA & TGC CTG TGT ACA CCC ACA CT & NM_001III302.I \\
\hline SAA & CCT GGG CTG CTA AAG TCA TC & AGG CCA TGA GGT CTG AAG TG & NM_001081853.I \\
\hline CCNI (CYR6I) & TCA GTC AGA GGG CAG ACC TT & CCC AAG TTA GGG AGG GAG AG & XM_00I495078.6 \\
\hline HIF-I $\alpha$ & TGA GCT TGC TCA TCA GTT GC & GCA ATT CAT CTG TGC CTT CA & XM_023627857.I \\
\hline TGF- $\beta_{1}$ & TCC TGG CGC TAC CTC AGT AAC & TGA CAT CAA AGG ACA GCC ATT C & HM_569606.1 ${ }^{44}$ \\
\hline TNF- $\alpha$ & GAG GGA AGA GCA GTT ACC GAA TG & GGC TAC AGG CTT GTC ACT TGG & NM_001081819.2 \\
\hline
\end{tabular}

*Primers were designed by one of the authors (LCB) unless otherwise indicated.

CYR6I = Cysteine-rich protein 6I. 